\title{
Anti-inflammatory and Cytotoxic Effect of Arrow Root Mediated Selenium Nanoparticles
}

\author{
Twinkle Francis, S Rajeshkumar*, Anitha Roy, T Lakshmi
}

\section{Twinkle Francis, S Rajeshkumar*, Anitha Roy, T Lakshmi}

Nanobiomedicine Lab, Department of Pharmacology, Saveetha Dental College and Hospital, SIMATS, Chennai - 600077, TN, INDIA.

\section{Correspondence \\ S Rajeshkumar}

Nanobiomedicine Lab, Department of Pharmacology, Saveetha Dental College and Hospital, SIMATS, Chennai -600077 , TN, INDIA.

E-mail: ssrajeshkumar@hotmail.com History

- Submission Date: 06-05-2020;

- Review completed: 01-07-2020;

- Accepted Date: 06-07-2020

DOI : 10.5530/pj.2020.12.188

Article Available online

http://www.phcogj.com/v12/i6

\section{Copyright}

(C) 2020 Phcogj.Com. This is an open access article distributed under the terms of the Creative Commons Attribution 4.0 International license.

\begin{abstract}
Nanoparticles serve to reduce toxicity, enhance bioactivity and improve targeting. Selenium nanoparticles (SeNPs) are explored because of it's unique characteristics and various known therapeutic benefits such as antioxidants activities and reducing risk of cancer. In this study, arrow root which is a starch obtained from tapioca or cassava is mediated with SeNPs and studied for it's anti-inflammatory and cytotoxic effect. The plant extract of arrow root was prepared and mixed with the sodium selenite solution. The solution is kept in a magnetic stirrer while being observed for any colour change. The synthesis of nanoparticles is observed using UV-vis spectrophotometry. Then the anti-inflammatory activity is observed using albumin denaturation assay and cytotoxic analysis conducted on brine shrimp nauplii. The results showed that there was a lower toxicity rate of SeNPs and significant anti-inflammatory activity which concludes that these nanoparticles can be used in various drug designing aspects in future.
\end{abstract}

Key words: Selenium nanoparticles, Green synthesis, Anti-inflammatory, Brine shrimp.

\section{INTRODUCTION}

Arrowroot scientifically known as Maranta arundinacea is a starch obtained from the rhizomes of several tropical plants such as Zamia integrifolia and tapioca from cassava. ${ }^{1}$ Arrowroot is used as a nutritional food for infants and also for people recovering from illness. It has a wide usage among stomach and intestinal disorders, including diarrhoea.

Medicines synthesised from arrow root are used in soothing painful gums and sore mouth by direct application onto the affected area. It is also known to cure athlete's foot skin infection which is a fungal infection of feet. ${ }^{2,3}$ In the food industry, arrowroot is used as an ingredient in cooking as a flavourless thickening agent.

Nanoparticles (NPs) are known to reduce toxicity, enhance bioactivity and also improve targeting. Among different nanoparticles such as silver nanoparticles and gold nanoparticles from inorganic metals, nanoparticles from selenium (Se) have been explored. Selenium is an essential nonmetal that has zero oxidation state which makes it a biologically inert material. ${ }^{4}$ Selenium has less therapeutic factors and delicate toxicity margins whereas the nanoparticles of selenium possess reduced toxicity and potential therapeutic benefits such as being an antioxidant, reducing risk of certain cancers, protection against heart diseases, etc. ${ }^{5,6}$ Selenium nanoparticles have been studied in various inflammation mediated disorders such as arthritis, cancer, diabetes and nephropathy. ${ }^{7}$ As an example of selenium nanoparticles function as a treatment beneficial to human health, some studies have shown that selenium nanoparticles can be taken in by cancer cells through endocytosis which induces apoptosis in cancer cells. ${ }^{8}$ Hence, functionalized selenium nanoparticles have become the leading innovation in controlling Se dosage for such human health benefits.

Studies have shown that selenium nanoparticles containing a variety of polysaccharides have a size range of 20-200 $\mathrm{nm}$ proving their benefit and hence demonstrating the importance

of using higher order saccharides to synthesise biologically therapeutic selenium nanoparticles. Using plants for NP synthesis can be advantageous over other biological processes because it eliminates the extensive process of cell culture maintenance and can also provide large scale NP synthesis. ${ }^{9,10}$ This research aims to provide conjunction between the immunological activity of arrow root and the antitumor activity of selenium nanoparticles materials in order to prove the anti inflammatory and cytotoxic activity of arrow root mediated with selenium nanoparticles.

\section{MATERIALS AND METHODS}

\section{Plant extracts preparation}

$1 \mathrm{~g}$ of arrow root powder was measured and taken. The measured amount of plant powder was then mixed with $100 \mathrm{ml}$ of distilled water and boiled for 5-10 mins. The contents were filtered

using a filter paper, funnel and measuring cylinder. A viscous filtrate was obtained (Figure 1).

\section{Selenium nanoparticles synthesis}

$30 \mathrm{mM}$ sodium selenite was prepared in $50 \mathrm{ml}$ of distilled water with $50 \mathrm{ml}$ of arrow root extract. Then the arrow root selenium solution was kept in the hot plate with magnetic stirrer for nanoparticle synthesis. The spectroscopy reading based on colour change was observed (Figure 2). 


\section{Albumin denaturation assay}

BSA (bovine serum albumin) was used as a reagent for the assay. BSA makes up approximately $60 \%$ of all proteins in animal serum hence is commonly used in cell culture.

$2 \mathrm{ml}$ of $1 \%$ bovine albumin fraction was mixed in different concentrations of arrow root selenium solution of $10 \mu \mathrm{I}, 20 \mu \mathrm{I}, 30 \mu \mathrm{I}$, $40 \mu \mathrm{I}, 50 \mu \mathrm{I}$ resp. and $\mathrm{pH}$ was adjusted to 6.8 using $1 \mathrm{~N} \mathrm{HCL}$. Then the reaction mixture was heated using a heating mantle at $37^{\circ} \mathrm{C}$ for $10 \mathrm{mins}$ in a water bath. Mixture is cooled and absorbance value was obtained at $660 \mathrm{~nm}$. Equal amount of plant extract was replaced with DMSO for control. Anti-inflammatory activity was analysed (Figure 3).

\section{Cytotoxicity analysis}

$3 \mathrm{~g}$ of sea salt was measured using an electronic weighing scale and mixed with $300 \mathrm{ml}$ of distilled water. A microplate was taken and 5 wells were marked as $10 \mu \mathrm{I}, 20 \mu \mathrm{I}, 30 \mu \mathrm{I}, 40 \mu \mathrm{I}, 50 \mu \mathrm{I}$ respectively along with one well marked as control. The wells were filled with the prepared salt solution and 10 nauplii are added to each well respectively. Arrowroot selenium is now taken in centrifuging tubes for centrifugation followed by heat treatment in the hot air oven. The nauplii was then added as 10 in number to each well using a transfer pipette. Then the prepared Se nanoparticles were added to each well with the respective measurement using the micropipette. The plate is kept undisturbed and observed after 12 hours (Figure 4 ).

\section{RESULTS}

\section{Formation of arrowroot mediated selenium} nanoparticles

In the performed selenium nanoparticles formulation process, sodium selenite solution was mixed with arrowroot and no chemical reaction was observed. Slight colour change was observed gradually in an interval of $24 \mathrm{~h}$. The UV-vis spectroscopic readings of arrow root selenium nanoparticles showed an absorbance value of $660 \mathrm{~nm} .^{11,12}$ The anti-inflammatory activity of Se NPs was studied using albumin denaturation assay. The graph obtained (Graph 1) showed a peak at different intervals.

\section{In vitro anti-inflammatory activity of arrow root mediated selenium nanoparticles}

A potential increase is seen in the anti-inflammatory property of arrow root mediated selenium nanoparticles in increasing concentration when compared to the standard diclofenac. The following calculations were done on the basis of the reading that was obtained (Graph 2).

\section{Calculation:}

\%Inhibition: Ac-As x 100

Ac

Where Ac is the absorbance of control and As is absorbance of sample.

\section{Cytotoxic activity of arrow root mediated selenium nanoparticles}

There were 60 nauplii in total with 10 nauplii distributed in each of the 5 wells including the control. In an interval of 12 hours, the effect of the selenium nanoparticles was observed. It was observed that only 5 nauplii were alive in the first well of the microplate with concentration $10 \mu \mathrm{I}, 3$ nauplii were alive in the second well with concentration 20 $\mu \mathrm{I}$ and 1 nauplii was alive in the third well with concentration $30 \mu \mathrm{I}$ (Graph 3).
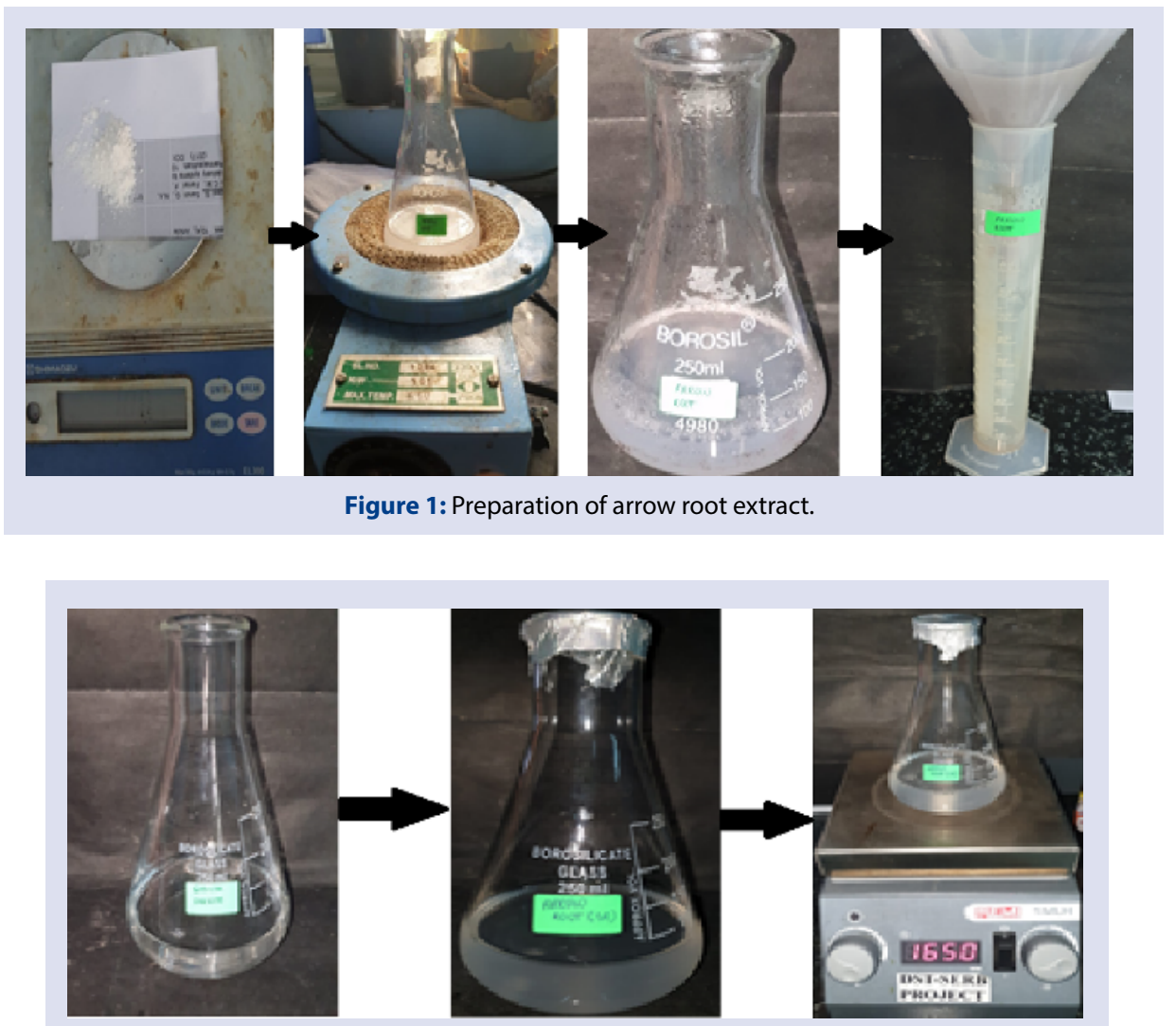

Figure 2: Preparation of arrow root selenium nanoparticles. 


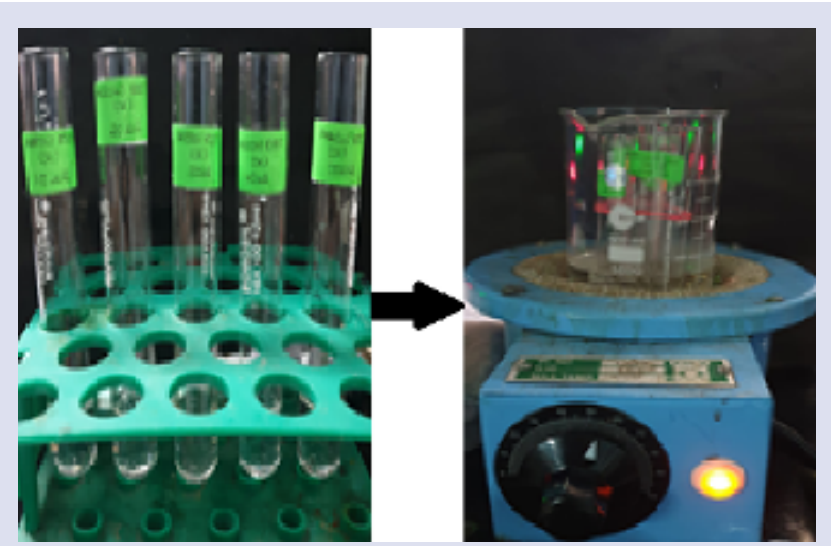

Figure 3: Albumin denaturation assay.

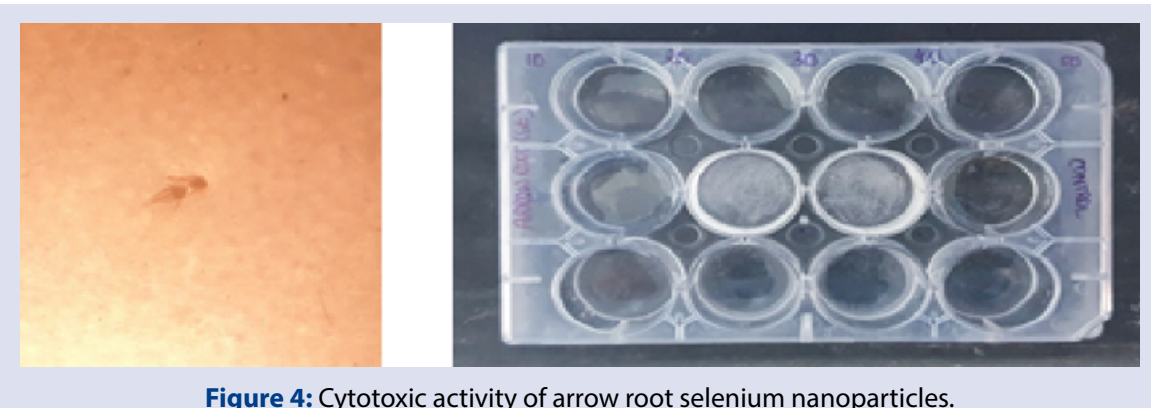

Figure 4: Cytotoxic activity of arrow root selenium nanoparticles.

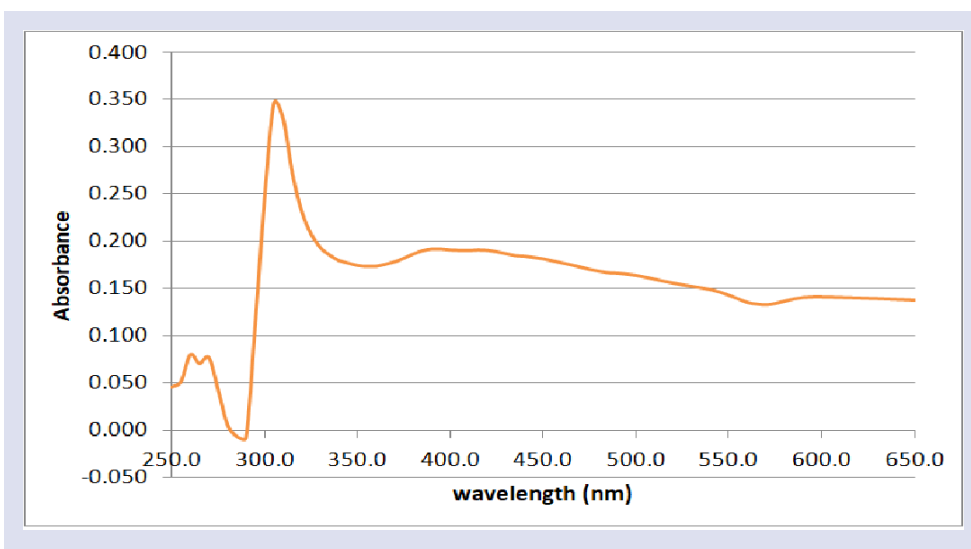

Graph 1: Spectroscopic analysis of selenium nanoparticles.

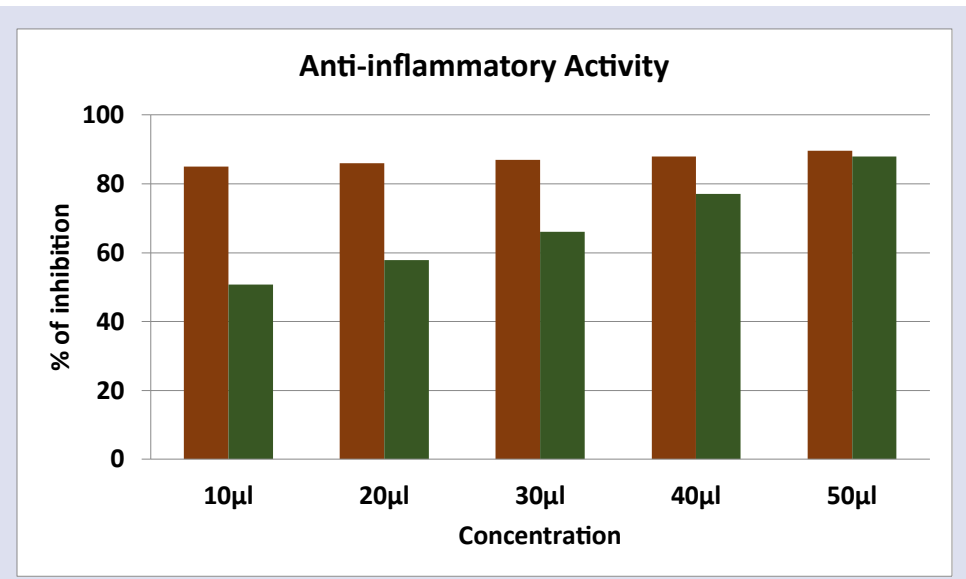

Graph 2: In-vitro anti-inflammatory activity of arrow root selenium nanoparticles. 


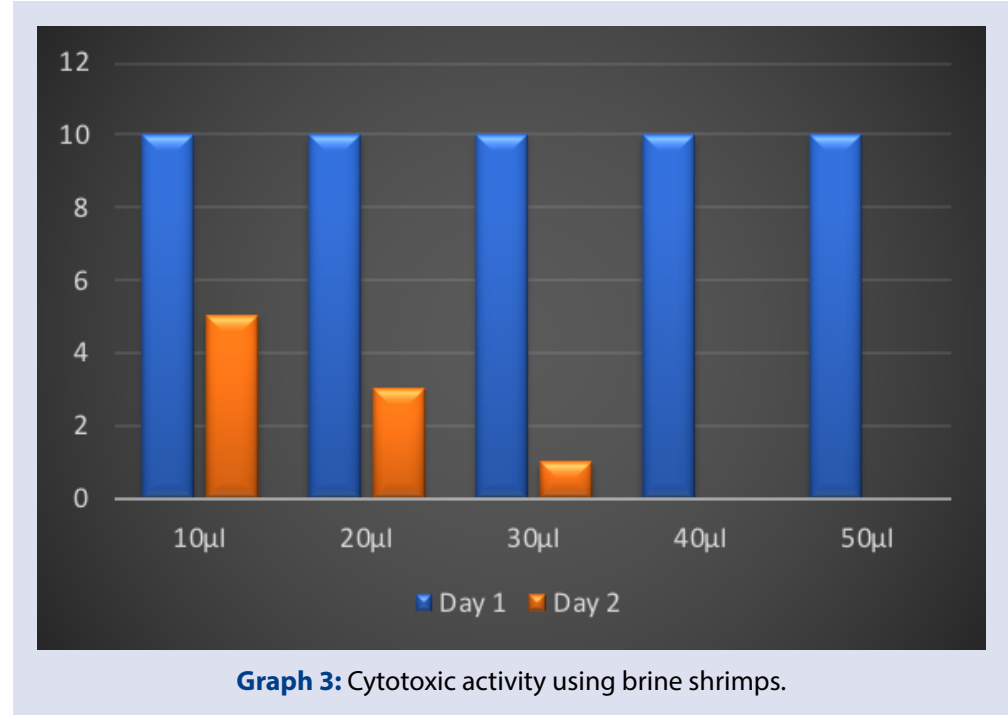

\section{DISCUSSION}

The characteristic absorption does not originate from any synthetic precursors because the solution was subjected to filtration to remove any impurities. The strongest absorption intensity hails from when arrowroot selenium NPs concentration increases. The graph obtained (Graph 1) showed a peak at different intervals indicating the synthesis of nanoparticles. ${ }^{11}$

A potential increase in the anti-inflammatory property of arrow root mediated selenium nanoparticles in increasing concentration when compared to the standard diclofenac shows that this formulation can be of good therapeutic benefit in case of inflammatory diseases. There has been a considerable increase in the exploration of nanoparticlebased therapeutics for the treatment of cancer, inflammation, allergy, infections and other oxidative stress related disorders. ${ }^{12}$

One article ${ }^{13}$ published in 2013 showed that it possesses antifungal activity due to its ability to cure Tinea Pedis and also has a glycemic index that is low, 32, and can be effective when used in a blood sugar diet. Arrow root comprises terpenoids which is what is known to prevent diseases like cancer and elicit properties such as immune modulation and anti-inflammation. This explains its usage in density as it adopts anti-inflammation mechanisms when penetrated into the mucous membrane of oral cavity and thereby suppressing swelling. ${ }^{14}$

The cytotoxic activity test of arrowroot mediated selenium nanoparticles indicates the decreased cytotoxic activity exhibited by the arrow root mediated selenium nanoparticles in lesser concentrations. Hence, this shows that dose dependent formulations of arrowroot mediated selenium nanoparticles may prove to be less toxic and safe for therapeutic treatments. ${ }^{15}$

\section{CONCLUSION}

This study proves that there are anti-inflammatory mechanisms adopted by the selenium nanoparticles. The arrowroot selenium nanoparticle formation is known to adopt cellular mechanisms to counteract and inhibit inflammation. Also, the confirmed lowered cytotoxic effect of arrow root mediated selenium nanoparticles provides a potential application of these in future. In future these nanoparticles can be used for new drug designing and targeting and offer treatment for various inflammatory disorders with minimal side effects. Also, these arrow root-stabilized nanoparticle formulations were demonstrated to have biocompatibility, as well as strong potential for application in the fields of medicine and food.

\section{REFERENCES}

1. Brito V, Godoy-Casagrande V, Narcisa-Oliveira J, Tomielis I, Cereda M, Steinfort $U$, et al. Phenological stages of arrowroot (Maranta arundinacea L.) according to the Biologische Bundesanstalt Bundessortenamt und Chemische Industrie scale [Internet]. Annals of Applied Biology. 2019;175:119-28.

2. Charles AL, Cato K, Huang TC, Chang YH, Ciou JY, Chang JS, et al. Functional properties of arrowroot starch in cassava and sweet potato composite starches. Food Hydrocolloids. 2016;53:187-91.

3. Harmayani E, Kumalasari ID, Marsono Y. Effect of arrowroot (Maranta arundinacea $\mathrm{L}$.) diet on the selected bacterial population and chemical properties of caecal digesta of Sprague Dawley rats. Int Res J Microbiol. 2011;2:278-84.

4. Soumya Menon, Shrudhi Devi KS, Santhiya R, Rajeshkumar S, Venkat Kumar S, Selenium nanoparticles: A potent chemotherapeutic agent and an elucidation of its mechanism. Colloids and Surfaces B: Biointerfaces. 2018;170:280-92.

5. Rajeshkumar S, Bharath LV. Mechanism of plant-mediated synthesis of silver nanoparticles - A review on biomolecules involved characterisation and antibacterial activity. Chemico-Biological Interactions. 2017;273:219-227.

6. H. Agarwal, S. Menon, S.V. Kumar, S. Rajeshkumar. Mechanistic study on antibacterial action of zinc oxide nanoparticles synthesized using green route. Chemico-Biological Interactions. 2018;286:60-70.

7. F. Gao, Q. Yuan, L. Gao, P. Cai, H. Zhu, R. Liu, et al. Cytotoxicity and therapeutic effect of irinotecan combined with selenium nanoparticles. Biomaterials. 2014;35:8854-66

8. H. Estevez, J.C. Garcia-Lidon, J.L. Luque-Garcia, C. Camara. Effects of chitosanstabilized selenium nanoparticles on cell proliferation, apoptosis and cell cycle pat- tern in HepG2 cells: Comparison with other selenospecies. Colloids Surf B. 2014;122:184-93.

9. S. Zhang, J. Zhang, H. Wang, H. Chen. Synthesis of selenium nanoparticles in the presence of polysaccharides. Mater Lett. 2004;58:2590-4.

10. X. Jia, Q. Liu, S. Zou, X. Xu, L. Zhang. Construction of selenium nanoparticles/ beta- glucan composites for enhancement of the antitumor activity. Carbohydr Polym. 2015;117:434-42.

11. J.A. Johnson, M.L. Saboungi, P. Thiyagarajan, R.A. Csencsits, D. Meisel, Selenium nanoparticles: a small-angle neutron scattering study. J Phys Chem B. 1999;103:59.

12. L.B. Yang, Y.H. Shen, A.J. Xie, J.J. Liang, B.C. Zhang. Synthesis of Se nanoparticles by using TSA ion and its photocatalytic application for decolorization of cango red under UV irradiation. Mater Res Bull. 2008;43:57282.

13. Hanani ZAN, Nur Hanani ZA, McNamara J, Roos YH, Kerry JP. Effect of plasticizer content on the functional properties of extruded gelatin-based composite films. 2013;31:264-9.

14. X. Jia, Q. Liu, S. Zou, X. Xu, L. Zhang. Construction of selenium nanoparticles/ beta- glucan composites for enhancement of the antitumor activity. Carbohyd Polym. 2015;117:434-42.

15. Y Xiao, Q. Huang, Z. Zheng, H. Guan, S. Liu. Construction of a Cordyceps sinensis exopolysaccharide-conjugated selenium nanoparticles and enhancement of their antioxidant activities. Int J Biol Macromol. 2017;99:483-91. 


\section{GRAPHICAL ABSTRACT}

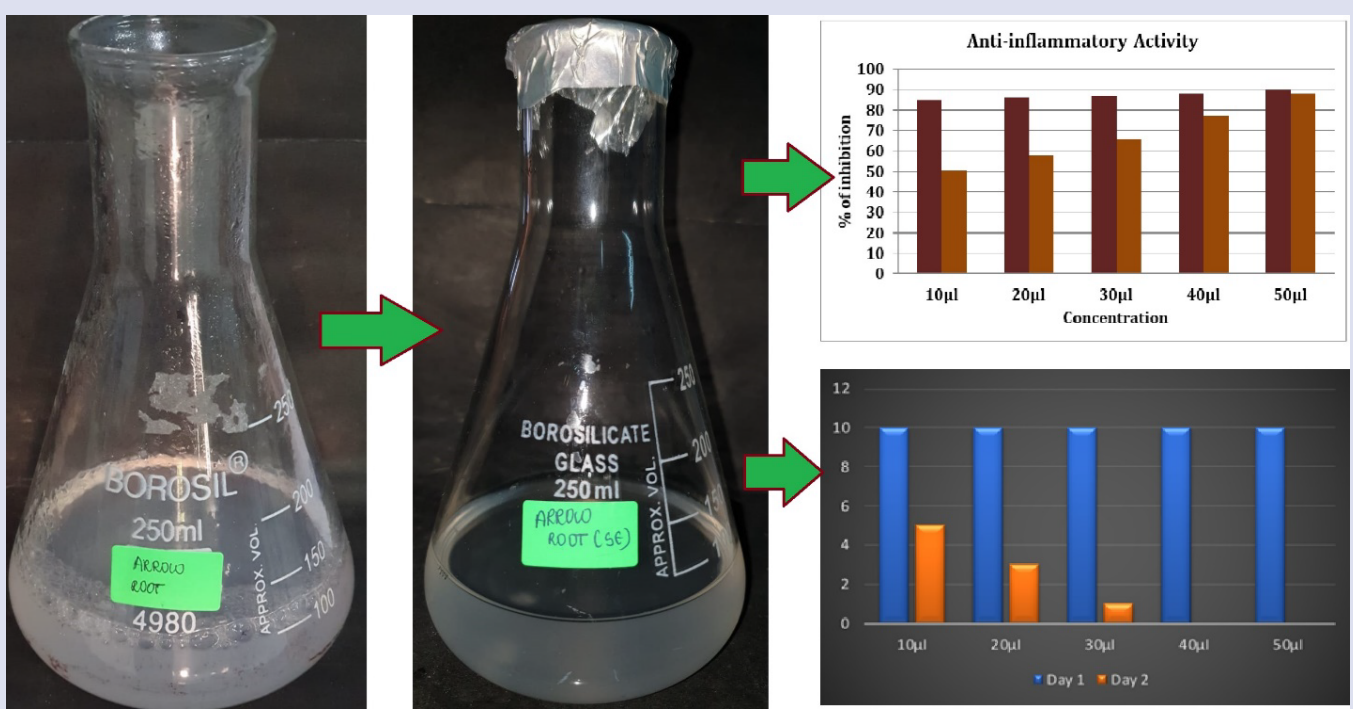

\section{ABOUT AUTHORS}

- Twinkle Francis is BDS Student and published 3 Articles.

- Dr. S Rajeshkumar is Associate Professor and Chief Scientist of Nano biomedicine Lab, Department of Pharmacology, SDC, SIMATS. He published more than 200 Research articles and 20 book chapters. He delivered Guest lecture in more than 30 Institutions. He reviewed many awards from National and International Conferences and Agencies.

- Dr. Anitha Roy is Associate Professor in Department of Pharmacology, SDC, SIMATS. And she published more than 150 research and review articles in International Journals.

- Dr. T Lakshmi is Associate Professor in Department of Pharmacology, SDC, SIMATS. And she published more than 200 research and review articles in International Journals.

Cite this article: Francis T, Rajeshkumar S, Roy A, Lakshmi T. Anti-inflammatory and Cytotoxic Effect of Arrow Root Mediated Selenium Nanoparticles. Pharmacogn J. 2020;12(6):1363-7. 